\title{
SIZE OF SUBSETS OF GROUPS AND HAAR NULL SETS
}

\author{
SŁAWOMIR SOLECKI
}

\begin{abstract}
This is a study of several notions of size of subsets of groups. The first part (Sections 3-5) concerns a purely algebraic setting with the underlying group discrete. The various notions of size considered there are similar to each other in that each of them assesses the size of a set using a family of measures and translations of the set; they differ in the type of measures used and the type of translations allowed. The way these various notions relate to each other is tightly and, perhaps, unexpectedly connected with the algebraic structure of the group. An important role is played by amenable, ICC (infinite conjugacy class), and FC (finite conjugacy class) groups.

The second part of the paper (Section 6), which was the original motivation for the present work, deals with a well-studied notion of smallness of subsets of Polish, not necessarily locally compact, groups-Haar null sets. It contains applications of the results from the first part in solving some problems posed by Christensen and by Mycielski. These applications are the first results detecting connections between properties of Haar null sets and algebraic properties (amenability, FC) of the underlying group.
\end{abstract}

\section{INTRODUCTION}

In the introduction, I will outline the main results of the paper starting with Sections 3-5. Before doing that, however, I will describe a couple of precursors to the notions of size of subsets of groups considered in these sections. The common underlying idea can be summarized as follows. A family of "test" measures on a group is fixed. A set $A$ is considered to be large if for each measure from the family there is a translate of $A$ with large measure; it is small if for some measure from the family all translates of $A$ have small measure.

An important role in the paper will be played by amenable groups. A locally compact group $G$ is called amenable if there exists a complex linear functional $m$ from the linear space of all (equivalence classes of) bounded, Haar measurable, complex valued functions on $G$ with the following two properties:

(i) $m(f) \geq 0$ for any real valued, non-negative $f$ and $m(\mathbf{1})=1$ where $\mathbf{1}$ is the function constantly equal to 1 ;

(ii) $m\left({ }_{g} f\right)=m(f)$ for any $f$ and any $g \in G$ where ${ }_{g} f(x)=f\left(g^{-1} x\right)$.

The theory of amenable groups is well developed. Good sources for it are [22] and [23].

2000 Mathematics Subject Classification. Primary: 22A10, 43A07; Secondary: 20F24, 28C10.

Key words and phrases. Amenable groups, FC groups, ICC groups, Haar null sets.

Research supported by NSF grant DMS-0102254.

To appear in GAFA. 
Mitchell, in his work characterizing subsets of amenable groups $G$ supporting a left invariant mean [17], considers a set $A$ to be large, left thick in his terminology, if each finite set is included in a right translate of $A$. In fact, by results of [17], these turn out to be precisely the sets for which there exists a left invariant mean $m$ with $m(A)=1$. The group $G$ is assumed discrete here. Later modifications of Mitchell's notion of largeness of a set were studied. For example, Day in [7] considers a set $A$ to be large, left lumpy in Day's terminology, if for each $\epsilon>0$ and each probability Borel measure, $A$ has a right translate of measure $>1-\epsilon$. The group $G$ is assumed locally compact here. For more on these notions see Day's survey paper [8].

This type of methods of measuring the size of subsets of groups (and functions on groups) come up in the proof of Paterson's characterization of amenable groups on which each left invariant mean is two-sided invariant [21] and in Emerson's characterization of amenability of locally compact groups [9].

The notion of Haar null sets, discovered in the theory of differentiation on separable Banach spaces, is another example of measuring the size of subsets of groups in the manner described above. A universally measurable subset $A$ of a group $G$ is considered small, Haar null, if for some Borel probability measure all two sided translates of $A, g A h$, have measure zero. The group is assumed to be Polish. Haar null sets were introduced by Christensen [4].

Obviously, there are two choices involved in the type of assessment of the size of subsets of groups I outlined above. The first one concerns the class of measures being used in the process. It is the family of all Borel probability measures in the case of left lumpy and Haar null sets and the family of all uniformly distributed probability measures with finite support in the case of left thick sets. The other choice concerns the type of translations being allowed. These are one sided (right) translations in the case of left thick and left lumpy sets and two sided translations in the case of Haar null sets.

In Sections 3-5, I study the relationships between the measures of size obtained by making these choices and the dependence of these relationships on the algebraic structure of $G$. These problems will be considered for discrete groups $G$. Originally, I investigated the questions of Sections $3-5$ in order to gain an understanding of measuring the size of sets needed to approach the problems on Haar null sets from Section 6. However, the results obtained are perhaps of independent interest. Moreover, Sections 4 and 5 have direct applications to the Haar null results.

From this point on, unless otherwise stated, all groups are considered with the discrete topology.

Two classes of measures on $G$ will be of interest to us. First the class of all probability measures. (By a probability measure on $G$ we mean a non-negative $l^{1}$ function on $G$ with the $l^{1}$ norm equal to 1.) The second class is that of uniformly distributed probability measures, that is, measures of the form $\mu_{F}(A)=|A \cap F| /|F|$ where $F \subseteq G$ is finite and non-empty.

For a probability measure $\mu$ on $G$ and $A \subseteq G$, set

$$
\mu^{L}(A)=\sup _{g \in G} \mu(g A)
$$


and similarly for two-sided translations let

$$
\mu^{T}(A)=\sup _{g, h \in G} \mu(g A h) .
$$

We can measure the size of $A \subseteq G$ by defining

$$
a(A)=\inf \left\{\mu^{L}(A): \mu \text { a probability measure on } G\right\},
$$

that is, $a(A)=\inf \left\{\left\|\mu * \chi_{A^{-1}}\right\|_{\infty}: \mu\right.$ is a probability measure $\}$. The question we address is whether all probability measures on $G$ need to be used as test measures to compute $a(A)$ and, in particular, if it suffices to use only uniformly distributed measures. Define

$$
u(A)=\inf \left\{\left(\mu_{F}\right)^{L}(A): F \subseteq G \text { finite and nonempty }\right\},
$$

or, in other words, $u(A)=\inf \left\{\left\|\mu_{F} * \chi_{A^{-1}}\right\|_{\infty}: F \subseteq G\right.$ finite non-empty $\}$. (Note that a set $A$ is right thick, see above, if, and only if, $u(A)=1$. Since $G$ is discrete, this is also equivalent to $A$ being right lumpy.) Now the question becomes: under what assumptions on $G$ do we have $a=u$ ? We define analogous quantities for two-sided translations by letting

$$
a^{T}(A)=\inf \left\{\mu^{T}(A): \mu \text { a probability measure on } G\right\}
$$

and

$$
u^{T}(A)=\inf \left\{\left(\mu_{F}\right)^{T}(A): F \subseteq G \text { finite and nonempty }\right\} .
$$

Again we ask under what circumstances $a^{T}=u^{T}$.

Below we formulate the main results in easy to state forms. Their sharper versions and proofs can be found in the appropriate sections.

A sharper version of the following theorem is proved in Section 4.

Theorem 1.1. Let $G$ be a group.

(i) If $G$ is amenable, then $a=u$

(ii) If $G$ contains a non-Abelian free group, then $a \neq u$. In fact, for each $\epsilon>0$ there exists $A \subseteq G$ with $u(A)>1-\epsilon$ and $a(A)<\epsilon$.

Rather surprisingly the analogous theorem for two-sided translations is very different. The following result is proved in Section 3.

Theorem 1.2. If $G$ is an arbitrary group, then $a^{T}=u^{T}$.

The proof of this result is perhaps unexpected. It starts with a short argument showing that there exists an amenable normal subgroup $H$ of $G$ such that the quotient group $G / H$ is ICC, that is, each non-identity element has infinite conjugacy class. Using a combination of combinatorial and measure theoretic methods, we prove next that a statement stronger than that in Theorem 1.2 holds for ICC groups. Then using amenability of the kernel of the quotient map $G \rightarrow G / H$, we transfer this stronger result from $G / H$ to $G$.

Combining Theorem 1.2 with the obvious inequalities, we get on an arbitrary group $G$

$$
a \leq u \leq u^{T}=a^{T} .
$$


When $a=u$ is explained to a large extent by Theorem 1.1. It remains, therefore, to analyze when the equality $u=u^{T}$ holds. If $G$ is a group, let

$$
G_{F C}=\left\{g \in G:\left\{h g h^{-1}: h \in G\right\} \text { is finite }\right\} .
$$

A straightforward calculation shows that $G_{F C}$ is a normal subgroup of $G$. A group is called $F C$ if $G_{F C}=G$, that is, if all elements of $G$ have finite conjugacy classes. FC groups are rather close to being Abelian, see [10], [19]. For example, it was proved in [19] that the center of a finitely generated FC group has finite index and that any finitely generated FC group can be mapped onto an Abelian group by a homomorphism with finite kernel.

A stronger form of the following result is established in Section 5.

Theorem 1.3. Let $G$ be a group.

(i) $u^{T} \leq\left[G: G_{F C}\right] u$.

(ii) There exists a set $A \subseteq G$ such that

$$
u^{T}(A)=1 \text { and } u(A)=\frac{1}{\left[G: G_{F C}\right]}
$$

where we interpret $1 /\left[G: G_{F C}\right]$ to be 0 if $\left[G: G_{F C}\right]$ is infinite.

In particular, $u^{T}=u$ if, and only if, $G$ is $F C$.

The second part of the paper deals with Haar null sets on Polish groups. Recall that a group is called Polish if it carries a separable completely metrizable group topology. All locally compact, second countable groups, their countable products, as well as all separable Banach spaces (with vector addition) are Polish groups. For other interesting examples see [1]. A subset of a Polish group $G$ is called Haar null if it is contained in a universally measurable set $A$ such that for some probability Borel measure $\mu$ on $G, \mu(g A h)=0$ for all $g, h \in G$. Recall that a subset of a Polish group is called universally measurable if it is measurable with respect to each Borel probability measure on the group. (The notion of Haar null sets has a curious history. Christensen, in [4] and [5], worked out the basic theory of it for Abelian Polish groups only, but he introduced the notion for arbitrary Polish groups [5, p.123]. Haar null sets on Abelian Polish groups were rediscovered by Hunt, Saur, and Yorke [12]. Basic results about Haar null sets for general Polish groups with their proofs were published by Hoffmann-Jørgensen [25] and, independently, by Mycielski [18].) Haar null sets are closed under taking countable unions and subsets. If $G$ happens to be locally compact, Haar null sets are precisely Haar measure zero sets. Therefore, the notion of Haar null sets can be viewed as extending the notion of Haar measure zero sets to not necessarily locally compact groups, that is, to groups on which left translation invariant, Borel, locally finite measures do not exist. This accounts for part of the interest in this notion. Its importance stems also from its applications in Banach spaces and Polish groups, for some examples of it see [5], [14], or [16]. For introductions to Haar null sets see [2], [5], and [25]. One should mention that there exist other useful measure theoretic notions of smallness in separable Banach spaces, see for example [2] and [15]. 
Haar null sets are understood quite well on locally compact groups and on Abelian Polish groups, in particular, on separable Banach spaces. It is not so without these topological or algebraic assumptions. This problem was already identified by Christensen in [5, p.122] but no results have been found so far indicating a connection between algebraic properties of non-locally compact Polish groups and properties of Haar null sets on them. We show that our investigation of sizes of subsets of discrete groups from Sections 3-5 can be used to understand Haar null sets on products of countable discrete groups. These groups are easy to handle topologically, yet they can be (and most of them are) non-locally compact and algebraically complicated, as complicated, in fact, as their factor groups. It turns out that the behavior of Haar null sets on product groups is strongly connected to algebraic properties of the factor groups and can be quite different from the Abelian and the locally compact cases.

Mycielski formulated two questions which make the problem of Christensen more precise. In [18], he asked if left Haar null sets, which are defined by modifying the definition of Haar null by allowing only left, rather than two sided, translations, coincide with Haar null sets. (Having two sided translations in the definition of Haar null is crucial in the proof showing that the union of two Haar null sets is Haar null; a very desirable property for small sets.) For Abelian and locally compact groups the answer is affirmative. Answering Mycielski's question, Shi and Thompson constructed in [24] a Borel subset $A$ of the Polish group of all homeomorphisms of the interval $[0,1]$ which is left Haar null but not Haar null. We find such an example in a group of the form $\prod_{n \in \mathbb{N}} H_{n}$ with all the $H_{n}$ 's countable and, in fact, we characterize, in terms of algebraic properties of the $H_{n}$ 's, the product groups for which such an example can be found.

Another question which was posed in [5, p.122] and [18] was whether, for a universally measurable subset $A$ of a Polish group which is not Haar null, 1 belongs to the interior of $A^{-1} A$. The interest in this property comes from the fact that its being true on a Polish group $G$ implies that $G$ has an automatic continuity property - any universally measurable homomorphism from $G$ to a second countable group is continuous. It is known to be true if $G$ is Abelian (a result due to Christensen [4], [5, Theorem 7.3]) or if $G$ is locally compact (a classical result going back to Steinhaus and Weil $[11,20.17])$ or if $A$ has the Baire property and is nonmeager (Pettis' theorem [5, Theorem 5.1]). We answer this question of Christensen and Mycielski in the negative. In fact, again we characterize those product groups for which this property holds.

Theorem 1.4. Let $H_{n}, n \in \mathbb{N}$, be countable groups. Consider the group $\prod_{n} H_{n}$ with the product topology. The following conditions are equivalent.

(i) A subset of $\prod_{n} H_{n}$ is left Haar null if, and only if, it is Haar null.

(ii) For each universally measurable subset $A$ of $\prod_{n} H_{n}$ which is not Haar null, 1 is in the interior of $A^{-1} A$.

(iii) All but finitely many of the $H_{n}$ 's are $F C$. 


\section{Notation}

We say that a measure is defined on a set when it is, in fact, defined on a $\sigma$ algebra of subsets of this set. We think of a measure $\mu$ on a set $X$ as a function on $l_{+}^{\infty}(\mu)$, the set of all non-negative bounded functions on $X$ which are $\mu$-measurable, and we write $\mu(v)$ for $\int_{X} v(x) d \mu(x)$ for $v \in l_{+}^{\infty}(\mu)$. If $\mu$ is a measure on a set $X$ and $f: X \rightarrow Y$ is a surjection, by $f_{*} \mu$ we mean the measure on $Y$ defined by $f_{*} \mu(v)=\mu(v \circ f)$. Most of the time we will study such functions defined on a group $G$ so $X=G$. For technical reasons, however, we will have to consider a more general situation when $X=Y \times G$. For a bounded function $v$ on $Y \times G$ and $g, h \in G$, let

$$
{ }_{g} v_{h}(y, x)=v\left(y, g^{-1} x h^{-1}\right), \quad{ }_{g} v(y, x)=v\left(y, g^{-1} x\right), \quad v_{h}(y, x)=v\left(y, x h^{-1}\right) .
$$

Now assume $Y$ and $G$ to be discrete. Let $\mu$ be a probability measure on $Y \times G$, that is, an assignment of weights to points of $Y \times G$, that is, an $l^{1}$ non-negative function on $Y \times G$ with the $l^{1}$ norm 1 . We let $S_{\mu}$ stand for the support set of $\mu$, that is, $\{g \in G: \mu(\{g\})>0\}$. We write $l_{+}^{\infty}(Y \times G)$ for the set of all bounded non-negative functions on $Y \times G$. All these functions are $\mu$-measurable. Sometimes we write $l_{+}^{\infty}$ if $Y$ and $G$ are obvious from the context. For a function $v \in l_{+}^{\infty}(Y \times G)$, let

$$
\mu^{T}(v)=\sup _{g, h \in G} \mu\left({ }_{g} v_{h}\right), \quad \mu^{L}(v)=\sup _{g \in G} \mu(g v), \text { and } \mu^{R}(v)=\sup _{h \in G} \mu\left(v_{h}\right) .
$$

These functions are no longer measures. However, they are homogeneous subadditive functions on $l_{+}^{\infty}$. For a set $A \subseteq Y \times G$, we write

$$
\mu^{T}(A)=\mu^{T}\left(\chi_{A}\right), \mu^{L}(A)=\mu^{L}\left(\chi_{A}\right), \text { and } \mu^{R}(A)=\mu^{R}\left(\chi_{A}\right) .
$$

where $\chi_{A}$ is the indicator function of $A$. Note that if $Y$ consists of one point, then, after naturally identifying $G$ with $Y \times G, \mu^{T}(A), \mu^{L}(A), \mu^{R}(A)$ coincide with so denoted quantities defined in the introduction. Given a measure $\mu$, we sometimes write ${ }_{g} \mu, \mu_{h}$, and ${ }_{g} \mu_{h}$ for measures defined on $l_{+}^{\infty}(Y \times G)$ by $\mu\left({ }_{g} v\right), \mu\left(v_{h}\right)$ and $\mu\left({ }_{g} v_{h}\right)$, respectively.

For two functions $\phi$ and $\psi$ defined on $l_{+}^{\infty}$, define

$$
\phi \leq \psi \Leftrightarrow\left(\phi(v) \leq \psi(v) \text { for all } v \in l_{+}^{\infty} \text { with }\|v\|_{\infty} \leq 1\right) .
$$

$|A|$ stands for the cardinality of $A$. We denote by $\mathbb{N}$ the set of all natural numbers (including 0 ).

\section{TWO-SIDED TRANSLATIONS}

We will formulate a theorem which is stronger than Theorem 1.2 and seems to represent the "right" generality for this result. Recall the definition (2.1) of $\leq$ which we will apply here to $\mu_{F}$ and $\mu^{T}$.

Theorem 3.1. Let $G$ be an arbitrary group and let $\mu$ be a probability measure on $G$. Given $\epsilon>0$ and $C>1$ there exists a finite non-empty subset $F$ of $G$ such that

$$
\mu_{F} \leq C \mu^{T}+\epsilon
$$


If we apply Theorem 3.1 to $\epsilon>0, C>1$, and the indicator function $\chi_{A}$ of a set $A \subseteq G$, then it immediately follows that $u^{T}(A) \leq C a^{T}(A)+\epsilon$. Since $\epsilon>0$ and $C>1$ are arbitrary, this is all that is needed to see that $a^{T}(A)=u^{T}(A)$ which is Theorem 1.2.

Before embarking on the proof of this result, we survey the classes of groups that will be used in it.

A group is $I C C$ if each non-identity element has infinite conjugacy class. ICC groups are of relevance in constructing examples of von Neumann algebras. For more on this and examples of such groups see [13, Section 6.7].

Recall from the introduction that a group is called FC if all its elements have finite conjugacy classes. The structure of such groups was thoroughly studied by Neumann in [19]. In particular, he observed that in a finitely generated FC group, the center has finite index. Thus, each such group is amenable and, since each group is a directed union of its finitely generated subgroups and amenable groups are closed under such unions, it follows that FC groups are amenable. We will use this fact below. (This result has been substantially generalized, see [23, Corollary 14.26].)

Day deduced from the fact that amenable groups are closed under directed unions and extensions by amenable groups that each group has a (unique) largest normal amenable subgroup [6, Lemma 1, p.518]. This subgroup is sometimes called the amenable radical and we will use this name here.

The proof of Theorem 3.1 constitutes the remainder of this section. We start with a simple observation which is however but pivotal to the proof.

Lemma 3.2. The quotient of a group by its amenable radical is ICC.

Proof. Let $G$ be a group and let $H$ be its amenable radical. Let $(G / H)_{F C}$ be the normal subgroup consisting of all elements in $G / H$ which have finite conjugacy classes. Then $(G / H)_{F C}$ is $\mathrm{FC}$ and, therefore, amenable. Thus, its preimage under the quotient map $G \rightarrow G / H$ is an amenable normal subgroup of $G$ containing $H$. Hence $(G / H)_{F C}$ consists of the identity only.

Lemma 3.2 will allow us to split the proof of Theorem 3.1 into two parts: one dealing with amenable groups and the other with ICC groups. We start with the amenable case.

Lemma 3.3. Let $H$ be an amenable group and let $Y$ be a set. Denote by $p$ the projection $Y \times H \rightarrow Y$. For any probability measures $\mu_{i}, i=1, \ldots, n$, on $Y \times H$ and $C>1$ there exists $K \subseteq H$ finite non-empty and such that for each $i \leq n$,

$$
p_{*} \mu_{i} \times \mu_{K} \leq C\left(\mu_{i}\right)^{L} .
$$

Proof. Let $S_{\mu_{i}}$, for $i=1, \ldots, n$, be the support of $\mu_{i}$ and let $S$ be the union of the projections of the $S_{\mu_{i}}$ 's on $H$. Find finite $K \subseteq H$ such that $\left|K S^{-1}\right|<C|K|$.

Fix now $i \leq n$. It will suffice to prove the inequality $v: Y \times H \rightarrow[0, \infty)$ with domain included in $p\left(S_{\mu_{i}}\right) \times K$. Define $A(v): Y \rightarrow \mathbb{R}$ by letting for $y \in Y$

$$
A(v)(y)=\sum_{x \in H} v(y, x)
$$


For any $v$ as above, we have

$$
\begin{aligned}
p_{*} \mu_{i}(A(v)) & =\sum_{(y, x) \in Y \times H} v(y, x) \sum_{h \in H} \mu_{i}(\{(y, h x)\}) \\
& =\sum_{h \in H}\left(\sum_{(y, x) \in Y \times H} v\left(y, h^{-1} x\right) \mu_{i}(\{(y, x)\})\right) \\
& =\sum_{h \in H} \mu_{i}\left({ }_{h} v\right) .
\end{aligned}
$$

Now if $v$ is equal to zero outside of $p\left(S_{\mu_{i}}\right) \times K$,

$$
p_{*} \mu_{i}(A(v))=|K|\left(p_{*} \mu_{i} \times \mu_{K}\right)(v)
$$

and, therefore, (3.1) gives

$$
\begin{aligned}
|K|\left(p_{*} \mu_{i} \times \mu_{K}\right)(v) & =\sum_{h \in H} \mu_{i}\left({ }_{h} v\right)=\sum\left\{\mu_{i}\left({ }_{h} v\right): S \cap h K \neq \emptyset\right\} \\
& \leq\left|S K^{-1}\right| \mu_{i}^{L}(v)<C|K| \mu_{i}^{L}(v) .
\end{aligned}
$$

Lemma 3.4. Let $G$ be a group, let $\mu$ be a measure on it, and let $C>1, \epsilon>0$. If $F \subseteq G$ is finite non-empty and such that $\mu_{F} \leq C \mu^{T}+\epsilon$, then for any $\epsilon^{\prime}>0$, there exists a finite set $B \subseteq G$ such that for any $v: G \rightarrow[0,1]$, there are $g, h \in B$ with

$$
\mu_{F}(v) \leq C \mu\left({ }_{g} v_{h}\right)+\epsilon+\epsilon^{\prime} .
$$

Proof. Note that for any positive number $\delta$, there is a finite family $\mathcal{A}$ of functions from $G$ to $[0,1]$ which are identically 0 outside of $F$ such that each function from $G$ to $[0,1]$ is uniformly approximated on $F$ up to $\delta$ by one of the functions from $\mathcal{A}$. Let $B$ be a finite collection of $g, h \in G$ which are needed to guarantee

$$
\mu_{F}(v) \leq C \mu\left({ }_{g} v_{h}\right)+\epsilon+\epsilon^{\prime} / 2 .
$$

for all functions $v$ in $\mathcal{A}$. Now let $v: G \rightarrow[0,1]$ and let $u \in \mathcal{A}$ uniformly approximate $v$ on $F$ up to $\delta>0$. Let $v^{\prime}$ be the function equal to $v$ on $F$ and to 0 outside of $F$. Then for some $g, h \in B$

$$
\begin{aligned}
\mu_{F}(v) & \leq \mu_{F}(u)+\delta \leq C \mu\left({ }_{g} u_{h}\right)+\epsilon+\epsilon^{\prime} / 2+\delta \\
& \leq C\left(\mu\left({ }_{g} v_{h}^{\prime}\right)+\delta\right)+\epsilon+\epsilon^{\prime} / 2+\delta \leq C \mu\left({ }_{g} w_{h}\right)+(C+1) \delta+\epsilon+\epsilon^{\prime} / 2 .
\end{aligned}
$$

By making $\delta$ appropriately small, we are done.

Lemma 3.5. Let $G$ be a group and $H$ an amenable normal subgroup of it. Assume that the conclusion of Theorem 3.1 holds for $G / H$. Then it holds for $G$ as well.

Proof. Let $G$ be a group with a probability measure $\mu$ on it. Fix $C>1$ and $\epsilon>0$. Let $H$ be an amenable normal subgroup of $G$. Denote by $\pi$ the projection $G \rightarrow G / H$. Combining our assumption that Theorem 3.1 holds for $G / H$ with Lemma 3.4 (applied to the group $G / H$ and the measure $\pi_{*} \mu$ ), we obtain a finite set $F^{\prime} \subseteq G / H$ and another finite set $B \subseteq G$ such that, for any $v^{\prime}: G / H \rightarrow[0,1]$ there are $g, h \in B$ with

$$
\mu_{F^{\prime}}\left(v^{\prime}\right) \leq C\left(\pi_{*} \mu\right)\left(_{\pi(g)} v_{\pi(h)}^{\prime}\right)+\epsilon .
$$


We will show that given $C^{\prime}>1$ there exists a finite set $F \subseteq G$ such that for any $v: G \rightarrow[0,1]$ there are $g, h \in B$ and $h_{0} \in H$ with

$$
\mu_{F}(v) \leq C \cdot C^{\prime} \cdot \mu\left({ }_{g h_{0}} v_{h}\right)+\epsilon .
$$

This will prove the lemma.

Let $Y \subseteq G$ be a selector of the family of cosets of $H$. Let $\phi: G \rightarrow G / H \times H$ be the bijection given by $\phi(g)=(\pi(g), h)$ where $h$ is the unique element of $H$ with $g=h y$ for a $y$ from $Y$. Now consider the finitely many measures on $G / H \times H$ given by $\phi_{*}\left(g \mu_{h}\right)$ for $g, h \in B$. By applying Lemma 3.3 to this family of measures and the constant $C^{\prime}$ we obtain a finite set $K \subseteq H$ such that for any $g, h \in B$ and any $v: G \rightarrow[0,1]$ there exists $h_{0} \in H$ such that

$$
\left(\pi_{*}\left({ }_{g} \mu_{h}\right) \times \mu_{K}\right)(v) \leq C^{\prime} \phi_{*}\left({ }_{g} \mu_{h}\right)\left(h_{0} v\right) .
$$

Define

$$
F=\bigcup_{i \leq n} K y_{i}
$$

where $y_{1}, \ldots, y_{n}$ are the elements of $Y$ which are mapped by $\pi$ to elements of $F^{\prime}$.

Let $v: G \rightarrow[0,1]$. Define $v^{\prime}: G / H \rightarrow[0,1]$ by letting it be 0 on cosets not containing any of the $y_{i}$ 's and

$$
v^{\prime}\left(\pi\left(y_{i}\right)\right)=\mu_{K y_{i}}\left(v\left\lceil H y_{i}\right)\right.
$$

for $i \leq n$. Note that the definition of $v^{\prime}$ gives

$$
\mu_{F}(v)=\mu_{F^{\prime}}\left(v^{\prime}\right)
$$

and

$$
g^{\prime}\left(\pi_{*} \mu\right)_{h^{\prime}}\left(v^{\prime}\right)=\left(g_{g^{\prime}}\left(\pi_{*} \mu\right)_{h^{\prime}} \times \mu_{K}\right)\left(v \circ \phi^{-1}\right)
$$

for $g^{\prime}, h^{\prime} \in G / H$. Formula (3.2) allows us to pick $\bar{g}, \bar{h} \in B$ such that

$$
\mu_{F^{\prime}}\left(v^{\prime}\right) \leq C_{\pi(\bar{g})}\left(\pi_{*} \mu\right)_{\pi(\bar{h})}\left(v^{\prime}\right)+\epsilon .
$$

Note that, for $g, h \in G$, we have

$$
\pi(g)\left(\pi_{*} \mu\right)_{\pi(h)}=\pi_{*}\left({ }_{g} \mu_{h}\right) .
$$

Combining (3.4), (3.5), (3.6), (3.7), we obtain that for

$$
\mu_{F}(v) \leq C\left(\pi_{*}\left(\bar{g}_{\bar{g}} \mu_{\bar{h}}\right) \times \mu_{K}\right)\left(v \circ \phi^{-1}\right)+\epsilon .
$$

Additionally, by (3.3), for some $h_{0} \in H$, we get

$$
\begin{aligned}
\left(\pi_{*}\left(\bar{g}_{\bar{g}} \mu_{\bar{h}}\right) \times \mu_{K}\right)\left(v \circ \phi^{-1}\right) & \leq C^{\prime}\left(\phi_{*}\left(\bar{g}_{\bar{g}} \mu_{\bar{h}}\right)\left(h_{0}\left(v \circ \phi^{-1}\right)\right)\right)=C^{\prime}{ }_{\bar{g}} \mu_{\bar{h}}\left(h_{0} v\right) \\
& =C^{\prime} \mu\left({ }_{\bar{g}} h_{0} v_{\bar{h}}\right) .
\end{aligned}
$$

By (3.8) and (3.9) we obtain for $h, g, h_{0}$ as specified above

$$
\mu_{F}(v) \leq C C^{\prime} \mu\left(\bar{g} h_{0} v_{\bar{h}}\right)+\epsilon
$$

which we were required to prove. 
We need a measure theoretic lemma. Here are some notions that will make the statement of the lemma more succinct. Let $X$ be a set. Let $v: X^{k} \rightarrow \mathbb{R}$. By a one-dimensional section of $v$ we mean a function of the form

$$
X \ni x \rightarrow v\left(x_{1}, \ldots, x_{i-1}, x, x_{i+1}, \ldots, x_{k}\right) \in \mathbb{R}
$$

for some fixed $1 \leq i \leq k$ and fixed $x_{1}, \ldots, x_{i-1}, x_{i+1}, \ldots, x_{k} \in X$. In the lemma below, the set $X$ comes equipped with a $\sigma$-algebra and all measures defined on this set or its products are defined on this $\sigma$-algebra or on its products, that is, the $\sigma$-algebras generated by the multi-dimensional cubes. When a function is defined on a product of some number of copies of $X$, it is said to be measurable if it is measurable with respect to the appropriate product $\sigma$-algebra. Note that under the convention adapted here all one dimensional sections of a measurable function are measurable.

Lemma 3.6. Let $\mu$ be a finite measure on $X$. Let $\mu_{0}$ be a probability measure on $X$. Assume that $\mu$ is absolutely continuous with respect to $\mu_{0}$.

Let $0<r<s$. For large enough $k \in \mathbb{N}$, any measurable $v: X^{k} \rightarrow \mathbb{R}$ with $0 \leq v \leq 1$ and $\int_{X^{k}} v d \mu_{0}^{k} \geq s$ has a measurable one-dimensional section whose integral with respect to $\mu$ is $\geq r \mu(X)$.

Proof. Let $s>r>0$ be given. Let $d \mu=f d \mu_{0}$ for some $\mu_{0}$-integrable function $f: X \rightarrow[0, \infty)$. Pick $\epsilon>0$ so that $(1-\epsilon)(s-\epsilon) \geq r$. Put

$$
A_{k}=\left\{\left(x_{1}, \ldots, x_{k}\right) \in X^{k}: \frac{1}{k} \sum_{i=1}^{k} f\left(x_{i}\right) \geq(1-\epsilon) \mu(X)\right\} .
$$

Since $\mu(X)=\int_{X} f(x) d \mu_{0}(x)$, by the Law of Large Numbers [3, pp.282-284], for large enough $k$, we have

$$
\mu_{0}^{k}\left(A_{k}\right) \geq 1-\epsilon
$$

Fix an $i$ between 1 and $k$. Let $s\left(x_{1}, \ldots, x_{i-1}, x_{i+1}, \ldots, x_{k}\right)$ be the integral with respect to $\mu$ of the one dimensional section of $v$ determined by the tuple $x_{1}, \ldots, x_{i-1}$, $x_{i+1}, \ldots, x_{k} \in X$. Using (3.10) and the facts that $\mu_{0}$ is a probability measure and $0 \leq v \leq 1$, we obtain

$$
\begin{aligned}
& \frac{1}{k} \sum_{i=1}^{k} \int_{X^{k-1}} s\left(x_{1}, \ldots, x_{i-1}, x_{i+1}, \ldots, x_{k}\right) d \mu_{0}^{k-1}\left(x_{1}, \ldots, x_{i-1}, x_{i+1}, \ldots, x_{k}\right) \\
& \quad=\frac{1}{k} \sum_{i=1}^{k} \int_{X^{k}} v\left(x_{1}, \ldots, x_{k}\right) f\left(x_{i}\right) d \mu_{0}^{k}\left(x_{1}, \ldots, x_{k}\right) \\
& \quad=\int_{X^{k}} v\left(x_{1}, \ldots, x_{k}\right)\left(\frac{1}{k} \sum_{i=1}^{k} f\left(x_{i}\right)\right) d \mu_{0}^{k}\left(x_{1}, \ldots, x_{k}\right) \\
& \quad \geq(1-\epsilon) \mu(X) \int_{A_{k}} v d \mu_{0}^{k} \\
& \quad \geq(1-\epsilon) \mu(X)(s-\epsilon) \geq r \mu(X) .
\end{aligned}
$$

The conclusion of the lemma follows. 
Lemma 3.7. Let $G$ be a group with a probability measure $\nu$ on it. Let $\mu$ be a probability measure on $G$. Assume that $\mu$ is absolutely continuous with respect to $\nu$. Let $g_{1}, g_{2}, \cdots \in G$. Then for any $s>r$ for $k \in \mathbb{N}$ large enough, we have that for any $v: G \rightarrow[0,1]$, if $\left(g_{1}^{-1} \nu * \cdots *_{g_{k}^{-1}} \nu\right)(v) \geq s$, then $\mu^{T}(v) \geq r$.

Proof. Fix $s>r$. Taking $X=G$ and applying Lemma 3.6 to $\mu_{0}=\nu$ and $\mu$, we find that for $k$ large enough the conclusion of the lemma holds. Fix such a $k$ and assume that $\left(g_{1}^{-1} \nu * \cdots *_{g_{k}^{-1}} \nu\right)(v) \geq s$ for some $v: G \rightarrow[0,1]$. Since ${ }_{g_{1}^{-1}} \nu * \cdots *_{g_{k}^{-1}} \nu=\phi_{*} \nu^{k}$, for the function $\phi: G^{k} \rightarrow G$ given by $\phi\left(h_{1}, \ldots, h_{k}\right)=g_{1} h_{1} \cdots g_{k} h_{k}$, it follows that $\nu^{k}(v \circ \phi) \geq s$. So a one-dimensional section of it has $\mu$-integral $\geq r$, that is, for some $h_{1}, \ldots, h_{i-1}, h_{i+1}, \ldots, h_{k} \in G$ we have

$$
\int_{G} v \circ \phi\left(h_{1}, \ldots, h_{i-1}, g, h_{i+1}, \ldots, h_{k}\right) d \mu(g) \geq r .
$$

However, the function

$$
G \ni g \rightarrow v \circ \phi\left(h_{1}, \ldots, h_{i-1}, g, h_{i+1}, \ldots, h_{k}\right)
$$

is simply equal to

$$
\left(g_{1} h_{1} \cdots g_{i-1} h_{i-1} g_{i}\right)^{-1} v_{\left(g_{i+1} h_{i+1} \cdots g_{k} h_{k}\right)^{-1}}
$$

which finishes the proof.

We will now prove two lemmas about ICC groups. The first one contains a result that is stronger than we need here. Its full strength will be used later on.

Lemma 3.8. Let $G$ be a group. Assume that $K_{1} \subseteq G$ is a finite set consisting of elements with infinite conjugacy classes. Then for any finite set $K_{2}$ the set $\left\{g \in G: g K_{1} g^{-1} \cap K_{2}=\emptyset\right\}$ has cardinality $|G|$.

Proof. For $g \in G, C(g)$ stands for the centralizer of $g:\left\{h \in G: h g h^{-1}=g\right\}$. Assume that the conclusion of the lemma fails. Let $K_{1}=\left\{h_{1}, \ldots, h_{n}\right\}$ and let $K_{2}=\left\{f_{1}, \ldots, f_{m}\right\}$ be such that, if we let

$$
B=\left\{g \in G: g K_{1} g^{-1} \cap K_{2} \neq \emptyset\right\},
$$

then $|G \backslash B|<|G|$. Note that

$$
B \subseteq \bigcup_{i \leq n, j \leq m} A_{i j}
$$

where $A_{i j}=\left\{g \in G: g h_{i} g^{-1}=f_{j}\right\}$. Let $S=\left\{(i, j): i \leq n, j \leq m, A_{i j} \neq \emptyset\right\}$. Now, if we pick $g_{i j} \in A_{i j}$ for $(i, j) \in S$, then an easy calculation shows that $g_{i j}^{-1} A_{i j} \subseteq C\left(h_{i}\right)$. Therefore, from (3.11), we get

$$
B \subseteq \bigcup_{(i, j) \in S} g_{i j} C\left(h_{i}\right)
$$

Since the complement of $B$ has cardinality smaller than $|G|$, there exists $g_{0} \in G$ with

$$
g_{0} \cdot(G \backslash B) \subseteq B
$$


Combining (3.12) and (3.13), we see that $G$ is covered by finitely many translates of $C\left(h_{i}\right), i=1, \ldots, n$. Now B.H. Neumann's lemma [20, Lemma 2.7] implies that there exists $i_{0} \leq n$ such that $C\left(h_{i_{0}}\right)$ has finite index in $G$. This is equivalent to $h_{i_{0}}$ having finite conjugacy class, contradiction.

Lemma 3.9. Let $G$ be an ICC group and let $P \subseteq G$ be finite. Then there exist $g_{1}, g_{2}, \cdots \in G$ such that, for any $k \in \mathbb{N}$, the mapping $\phi: P^{k} \rightarrow G$ given by $\phi\left(h_{1}, \ldots, h_{k}\right)=g_{1} h_{1} g_{2} h_{2} \cdots g_{k} h_{k}$ is injective.

Proof. We choose $g_{1}, g_{2}, \cdots \in G$ recursively as follows. Let $Q=P P^{-1} \backslash\{1\}$. We require that for each $i>1$,

$$
\left(\left(g_{1} P g_{2} P \cdots g_{i-1} P\right)^{-1} g_{1} P g_{2} P \cdots g_{i-1} P\right) \cap g_{i} Q g_{i}^{-1}=\emptyset .
$$

Note that these conditions are easily fulfilled by Lemma 3.8 since $G$ is ICC. We claim that with this choice of $g_{i}$ 's, given $k, \phi$ defined as in the statement of the lemma is 1-to-1. Let $\left(h_{1}, \ldots, h_{k}\right)$ and $\left(h_{1}^{\prime}, \ldots, h_{k}^{\prime}\right)$ be different sequences from $P^{k}$ and let $i_{0} \leq k$ be the largest $i$ with $h_{i} \neq h_{i}^{\prime}$. If $\phi$ applied to these two sequence yielded the same value, we would have

$$
g_{1} h_{1} g_{2} \cdots g_{i_{0}} h_{i_{0}}=g_{1} h_{1}^{\prime} g_{2} \cdots g_{i_{0}} h_{i_{0}}^{\prime}
$$

which if $i_{0}=1$, would give $h_{1}=h_{1}^{\prime}$, a contradiction with our choice of $i_{0}$, and if $i_{0}>1$,

$$
\left(g_{1} h_{1}^{\prime} g_{2} \cdots h_{i_{0}-1}^{\prime}\right)^{-1} g_{1} h_{1} g_{2} \cdots h_{i_{0}-1}=g_{i_{0}} h_{i_{0}}^{\prime} h_{i_{0}}^{-1} g_{i_{0}}^{-1}
$$

which in turn would contradict (3.14).

Proof of Theorem 3.1. By Lemmas 3.2 and 3.5, it remains to show that the conclusion of Theorem 3.1 holds for all ICC groups. In fact, we will show that it holds with $C=1$. Fix $\epsilon>0$.

We apply Lemma 3.9 to obtain a sequence $g_{1}, g_{2}, \cdots \in G$ so that for each $k$ the function $\phi_{k}: P^{k} \rightarrow G$ defined, as in the lemma, by

$$
\phi_{k}\left(h_{1}, \ldots, h_{k}\right)=g_{1} h_{1} g_{2} h_{2} \cdots g_{k} h_{k}
$$

is 1 -to- 1 .

We assume that the support $P$ of $\mu$ is finite. Let $\nu=\mu_{P}$. Clearly $\mu$ is absolutely continuous with respect to $\nu$. Therefore, we are in a situation in which we can apply Lemma 3.7 we only need to specify a pair of positive reals $r<s$. Fix $M \in \mathbb{N}$ so that $1 / M<\epsilon / 2$. Let $j$ be an integer with $0<j<M$. For each pair of the form $r=j / M$ and $s=(j+1) / M$, the conclusion of the lemma holds for $k$ large enough. So we can find a $k$ for which this conculsion holds for all $r, s$ of the above form. It follows then easily that for any $v: G \rightarrow[0,1]$,

$$
\left({ }_{g_{1}^{-1}} \nu * \cdots * g_{k}^{-1} \nu\right)(v) \leq \mu^{T}(v)+\epsilon .
$$

Since $g_{1}^{-1} \nu * \cdots *_{g_{k}^{-1}} \nu=\left(\phi_{k}\right)_{*} \nu^{k}$ and since $\nu$ is a normalized counting measure and $\phi_{k}$ is 1 -to-1, it follows that

$$
g_{1}^{-1} \nu * \cdots *_{g_{k}^{-1}} \nu=\mu_{D}
$$


where $D=\phi_{k}\left(P^{k}\right)$. Now taking into account (3.15), we get

$$
\mu_{D}(v)<\mu^{T}(v)+\epsilon
$$

which proves the theorem.

\section{LEFT tRANSLATIONS}

Theorem 1.1 follows immediately from the following result.

Theorem 4.1. Let $G$ be a group.

(i) If $G$ is amenable, then, for $C>1$, for each probability measure $\mu$ there exists a finite non-empty set $F$ such that

$$
\mu_{F} \leq C \mu^{L} .
$$

(ii) If $F_{2}<G$, then, for any $\epsilon>0$, there exists $A \subseteq G$ with $u(A)>1-\epsilon$ and $a(A)<\epsilon$.

Proof. (i) This is simply Lemma 3.3 for $Y$ a one point set and all the $\mu_{i}$ 's equal to $\mu$.

(ii) Fix $\epsilon>0$, and let $c=1-\epsilon$. Put $\sigma_{n}=\sum_{i=1}^{n} \frac{1}{i}$. Fix $n$ so that

$$
-\ln \epsilon+3<\epsilon \sigma_{n} .
$$

Since the free group on two generators contains $F_{n}$, the free group on $n$ generators, $F_{n}$ is a subgroup of $G$. Let $b_{1}, b_{2}, \ldots, b_{n}$ be free generators of $F_{n}$. Let $\nu$ be a probability measure whose support is $S_{\nu}=\left\{b_{j}: j \leq n\right\}$ and which is defined there by

$$
\nu\left(\left\{b_{j}\right\}\right)=\frac{1}{\sigma_{n} \cdot j} .
$$

For each finite set $D \subseteq F_{n}$ we will define now $D^{\prime} \subseteq D$ with the properties spelled out in (4.4) and (4.5). Fix $s \in F_{n}$ written in the reduced form. Define $D_{s}$ to consist of all the generators $b_{j}$ such that $s$ does not end in $b_{j}^{-1}$ and $s b_{j} \in D$. Further, let $m_{s} \in \mathbb{N}$ be such that

$$
c\left|D_{s}\right| \leq\left|\left\{b_{j}: b_{j} \in D_{s}, j \geq m_{s}\right\}\right|<c\left|D_{s}\right|+1 .
$$

Let

$$
D^{\prime}=\left(D \backslash \bigcup_{s \in F_{n}} s D_{s}\right) \cup \bigcup_{s \in F_{n}} s\left\{b_{j} \in D_{s}: j \geq m_{s}\right\} .
$$

Note that $s_{1} D_{s_{1}} \cap s_{2} D_{s_{2}}=\emptyset$, hence, by the first inequality in (4.2), $D^{\prime}$ contains at least $c\left|\bigcup_{s \in F_{n}} s D_{s}\right|$ of the elements of $\bigcup_{s \in F_{n}} s D_{s}$. From this we immediately get

$$
\left|D^{\prime}\right| \geq c|D| \text {. }
$$

We will now show that for any $s \in F_{n}$, we have

$$
\nu\left(s^{-1} D^{\prime}\right)<\epsilon .
$$


There is at most one $1 \leq j \leq n$ with $s$ ending in $b_{j}^{-1}$ and, for all but this one $j$, $s b_{j} \in D^{\prime}$ implies $b_{j} \in D_{s}$ and $j \geq m_{s}$. Thus, using (4.2) we get

$$
\begin{aligned}
\nu\left(s^{-1} D^{\prime}\right) & \leq \frac{1}{\sigma_{n}} \sum_{s b_{j} \in D^{\prime}} \frac{1}{j} \leq \frac{1}{\sigma_{n}}+\frac{1}{\sigma_{n}} \sum\left\{\frac{1}{j}: j \geq m_{s}, b_{j} \in D_{s}\right\} \\
& \leq \frac{1}{\sigma_{n}}+\frac{1}{\sigma_{n}}+\frac{1}{\sigma_{n}} \sum\left\{\frac{1}{j}: m_{s} \leq j \leq m_{s}+c\left|D_{s}\right|\right\}
\end{aligned}
$$

Now, the second inequality in (4.2) implies $\left|D_{s}\right|-\left(m_{s}-1\right)<c\left|D_{s}\right|+1$, which by a simple calculation gives $(1-c)^{-1} m_{s} \geq m_{s}+c\left|D_{s}\right|$. Applying it to (4.6) and using (4.1), we obtain

$$
\begin{aligned}
\nu\left(s^{-1} D^{\prime}\right) & \leq \frac{2}{\sigma_{n}}+\frac{1}{\sigma_{n}} \sum\left\{\frac{1}{j}: m_{s} \leq j \leq \frac{m_{s}}{1-c}\right\} \\
& \leq \frac{2}{\sigma_{n}}+\frac{1}{\sigma_{n}}+\frac{1}{\sigma_{n}} \int_{m_{s}}^{m_{s}(1-c)^{-1}} \frac{1}{x} d x \leq \frac{-\ln (1-c)+3}{\sigma_{n}}<\epsilon
\end{aligned}
$$

and (4.5) is proved.

Let $Y \subseteq G$ be a selector of the family of all the left cosets of $F_{n}$ in $G$. Let $P \subseteq G$ be a finite set. Define

$$
P^{*}=\bigcup_{g \in Y}\left(g^{-1} P \cap F_{n}\right)^{\prime}
$$

where the operation $(\cdot)^{\prime}$ on the right hand side of the above formula is defined by (4.3). It follows immediately from (4.4), (4.5), and the fact that the support of $\nu$ is contained in $F_{n}$ that for any finite $P \subseteq G$

$$
\left|P^{*}\right| \geq c|P|
$$

and

$$
\nu\left(g P^{*}\right)<\epsilon \text { for all } g \in G .
$$

Let $P_{i}, i<|G|$, list all finite subsets of $G$. By transfinite recursion, we easily find elements $g_{i} \in G, i<|G|$, so that for $i<j$ we have

$$
g_{j} \notin \bigcup_{i<j} g_{i} P_{i} S_{\nu}^{-1} S P_{j}^{-1} .
$$

This equation is set up to guarantee that for $i \neq j$ the sets $g_{i} P_{i} S_{\nu}^{-1}$ and $g_{j} P_{j} S_{\nu}^{-1}$ are disjoint which immediately implies that

$$
\forall g \in G S_{\nu} \cap g g_{i} P_{i} \neq \emptyset \text { for at most one } i<|G| \text {. }
$$

Define now

$$
A=\bigcup_{i<|G|}\left(g_{i} P_{i}\right)^{*}
$$

where the operation $(\cdot)^{*}$ is described by $(4.7)$.

Note that if $F \subseteq G$ is finite non-empty then $F=P_{i}$ for some $i<|G|$ and, therefore, by (4.8),

$$
\mu_{F}\left(g_{i}^{-1} A\right) \geq \mu_{P_{i}}\left(g_{i}^{-1}\left(g_{i} P_{i}\right)^{*}\right)>c
$$


On the other hand, from (4.10) and (4.9) we get that, for any $g \in G, \nu(g A)<\epsilon$. Thus, $A$ is as required.

Remark. For countable $G$, point (ii) in Theorem 4.1 can be strengthened as follows. For each $n=1,2, \ldots$, we introduce an auxiliary measure of sizes of subsets of $G$. Recall that $\sigma_{n}=\sum_{i=1}^{n} \frac{1}{i}$. For $v: G \rightarrow[0,1]$ define

$$
a_{n}(v)=\sigma_{n} \inf \left\{\mu^{L}(v): \mu \text { a probability measure on } G \text { with }\left|S_{\mu}\right| \leq n\right\} .
$$

The following statement justifies the introduction of the quantity $a_{n}$ :

$$
a \leq u \leq a_{n} \text { for each } n \in \mathbb{N} .
$$

Here is its proof. $a \leq u$ is obvious. To show $u \leq a_{n}$, it will suffice to prove that for any probability measure $\mu$ with $\left|S_{\mu}\right|=n$, there exists a finite nonempty set $F$ such that for any $v: G \rightarrow[0,1]$,

$$
\mu_{F}(v) \leq \sigma_{n} \mu(v)
$$

Let $g_{1}, \ldots, g_{n}$ list $S_{\mu}$ in such a way that $\mu\left(\left\{g_{i}\right\}\right) \geq \mu\left(\left\{g_{i+1}\right\}\right)$. Put $w_{i}=\mu\left(\left\{g_{i}\right\}\right)$. Let $k \leq n$ be such

$$
k w_{k}=\max \left\{i w_{i}: i=1, \ldots, n\right\} .
$$

We claim $F=\left\{g_{1}, \ldots, g_{k}\right\}$ does the job. Since $i w_{i} \leq k w_{k}$ for all $i=1, \ldots, k$, we have $1=\sum_{i=1}^{n} w_{i} \leq k w_{k} \sum_{i=1}^{n} 1 / i$ whence

$$
\frac{1}{k} \leq w_{k} \sigma_{n} .
$$

Now let $v: G \rightarrow[0,1]$. It follows from (4.12) and the fact that the $w_{i}$ 's are nonincreasing that

$$
\mu_{F}(v)=\frac{1}{k} \sum_{i=1}^{k} v\left(g_{i}\right) \leq \sigma_{n} \sum_{i=1}^{k}\left(v\left(g_{i}\right) w_{k}\right) \leq \sigma_{n} \sum_{i=1}^{k} v\left(g_{i}\right) w_{i} \leq \sigma_{n} \mu(v) .
$$

Now Theorem 4.1(ii) can be strengthened to the following.

If $G$ is countable and $F_{2}<G$, then, for each $\epsilon>0$ there exists $A \subseteq G$ with $u(A) \geq 1-\epsilon$ and $a_{n}(A) \leq-\ln \epsilon+3$ for each $n$.

Note that the condition $a_{n}(A) \leq-\ln \epsilon+3$ for each $n$ implies that $a(A) \leq$ $(-\ln \epsilon+3) / \sigma_{n}$ for each $n$; thus, $a(A)=0$.

We will leave it to the reader to modify the proof of Theorem 4.1 to establish the statement above.

\section{LEFT AND TWO-SIDED TRANSLATIONS}

Theorem 1.3 is an immediate consequence of the following result. To state this result in full strength, let us introduce one more piece of notation. For a subset $A$ of a group $G$, let $u^{R}(A)$ be defined as $u(A)$ except that the right, rather than left, translations of $A$ are taken in the definition. Clearly, $u^{T} \geq u^{R}$.

Point (i) of the theorem below gives $u^{T} \leq\left[G: G_{F C}\right] a$ and hence also $u^{T} \leq[G$ : $\left.G_{F C}\right] u$. Point (ii) shows that this estimate cannot be improved.

Theorem 5.1. Let $G$ be a group, and let $G_{F C}$ be the normal subgroup of $G$ consisting of all finite conjugacy class elements. 
(i) Let $C>1$ and let $\mu$ be a probability measure on $G$. There exists a finite set $K \subseteq G$ such that

$$
\left(\mu_{K}\right)^{T} \leq C \cdot\left[G: G_{F C}\right] \cdot \mu^{L} .
$$

(ii) If $\left[G: G_{F C}\right]$ is finite let $p=\left[G: G_{F C}\right]$; if $\left[G: G_{F C}\right]$ is infinite, let $p$ be any natural number. There exist $A \subseteq G$ and $D \subseteq G$ with $|D|=p$ such that

(a) $u^{R}(A)=1$;

(b) $h A \cap D$ has at most one element for any $h \in G$; in particular, $u(A) \leq$ $1 / p$.

Lemma 5.2. Let $G$ be a group with $M=\left[G: G_{F C}\right]$ finite and let $Y$ be a set. Denote by $p$ the projection from $Y \times G$ to $Y$. For any probability measure $\mu$ on $Y \times G$ and $C>1$ there exists a finite non-empty set $F \subseteq G$ such that

$$
\left(p_{*} \mu \times \mu_{F}\right)^{T}<C M \mu^{L} .
$$

Proof. The assumption that $M$ is finite implies that $G$ is amenable. Thus, by Lemma 3.3, it will suffice to prove our lemma only for a measure $\mu$ of the form $\nu \times \mu_{F}$ for a finite non-empty subset $F$ of $G$ and a probability measure $\nu$ on $Y$. This boils down to showing that for any $F \subseteq G$ finite and non-empty and any $C>1$ there exists a $K \subseteq G$ finite non-empty with $\left(\mu_{K}\right)^{T} \leq C M\left(\mu_{F}\right)^{L}$. Fix, therefore, $F$ and a constant $C>1$. Put $H=G_{F C}$. Let $\left\{g_{1}, \ldots, g_{M}\right\}$ select one element from each coset of $H$ in $G$. Since conjugacy classes in $G$ of elements of $H$ are finite and included in $H$, the set

$$
L=\bigcup_{i=1}^{M} \bigcup_{g \in G} g^{-1}\left(g_{i}^{-1} F \cap H\right) g
$$

is a finite subset of $H$. Since $H$, being FC, is amenable, we can find a finite set $K \subseteq G$ such that $\left|L K^{-1}\right| \leq C|K|$.

Now let $v: G \rightarrow[0,1]$ be given. Note that it will suffice to show that for any $f \in G$,

$$
\mu_{K}\left(v_{f}\right) \leq C M\left(\mu_{F}\right)^{L}(v)
$$

Fix, therefore, $f \in G$. Define

$$
F^{\prime}=\bigcup_{i=1}^{M} g_{i} f^{-1}\left(g_{i}^{-1} F \cap H\right) f .
$$

Note that since $H$ is normal, $|F|=\left|F^{\prime}\right|$. Now we compute

$$
\begin{aligned}
|K| \mu_{K}\left(v_{f}\right) & =\sum_{x \in K} v_{f}(x)=\sum_{g \in G} \mu_{F^{\prime}}\left(g\left(v_{f} \mid K\right)\right) \\
& =\frac{1}{\left|F^{\prime}\right|} \sum_{g \in G} \sum_{i=1}^{M} \sum\left\{\left(\left(g_{g} v_{f}\right) \mid g K\right)(x): x \in g_{i} f^{-1}\left(g_{i}^{-1} F \cap H\right) f\right\} \\
& \leq \frac{1}{|F|} \sum_{i=1}^{M} \sum_{g \in\left(g_{i} f^{-1}\left(g_{i}^{-1} F \cap H\right) f\right) K^{-1}} \sum\left\{\left({ }_{g} v\right)(x): x \in g_{i} f^{-1} g_{i}^{-1} F\right\}
\end{aligned}
$$


and we continue after substituting $y=g_{i} f g_{i}^{-1} x$ and noticing that, for each $0 \leq i \leq$ $M, f^{-1}\left(g_{i}^{-1} F \cap H\right) f \subseteq L$

$$
\begin{aligned}
& \leq \frac{1}{|F|} M\left|L K^{-1}\right| \max _{i \in\{1, \ldots, M\}} \max _{g \in g_{i} L K^{-1}} \sum\left\{\left(_{g_{i} f g_{i}^{-1} g} v\right)(y): y \in F\right\} \\
& \leq C M|K|\left(\mu_{F}\right)^{L}(v) .
\end{aligned}
$$

Thus, (5.1) follows, and we are done.

Proof of Theorem 5.1. (i) This is a special case of Lemma 5.2.

(ii) The proof of this point is a refinement of the proof of Lemma 3.1 in [21]. Put $M=\left[G: G_{F C}\right]$. Let $a_{1}, \ldots, a_{p}, p \in \mathbb{N}$, be elements of $G$ chosen from different cosets of $G_{F C}$. If $M$ is finite, we let $p$ be equal to $M$; if $M$ is infinite, $p$ is an arbitrary natural number. Enumerate $K_{\alpha}, \alpha<|G|$, finite subsets of $G$. By transfinite induction we construct $A_{\alpha} \subseteq G$ and $h_{\alpha} \in G, \alpha<|G|$, so that

(1) $A_{\alpha}=\bigcup_{\beta \leq \alpha} K_{\beta} h_{\beta}$;

(2) $a_{j}^{-1} a_{i} \notin A_{\alpha}^{-1} A_{\alpha}$ if $i \neq j, i, j \leq p$.

Assuming this has been accomplished, we define $A=\bigcup_{\alpha<|G|} A_{\alpha}$. Clearly (1) implies that $u^{R}(A)=1$. On the other hand, conditions (1) and (2) imply that any left translate of the set $D=\left\{a_{1}, \ldots, a_{p}\right\}$ intersects $A$ in at most one point. Therefore, $\mu_{D}^{L}(A)=1 / p$ implying that $u(A) \leq 1 / p$.

To define the sequence with properties (1) and (2), let $A_{-1}=\emptyset$. Assume $A_{\alpha^{\prime}}$ and $h_{\alpha^{\prime}}$, for $\alpha^{\prime}<\alpha$, have been constructed. Let $B=\bigcup_{\alpha^{\prime}<\alpha} A_{\alpha^{\prime}}$ and let $L=$ $\left\{a_{j}^{-1} a_{i}: i \neq j, i, j \leq p\right\}$. Since the conjugacy classes of all elements $a_{j}^{-1} a_{i}, i \neq j$, are infinite, by Lemma 3.8, the set of all $h \in G$ such that $h L h^{-1} \cap K_{\alpha}^{-1} K_{\alpha}=\emptyset$ has cardinality $|G|$. That is, there is a subset of $G$ of cardinality $|G|$ consisting of $h$ such that for all $i \neq j$,

$$
a_{j}^{-1} a_{i} \notin h^{-1} K_{\alpha}^{-1} K_{\alpha} h .
$$

Since, by our inductive assumption (1), $B$ has cardinality smaller than $|G|$, there exists an $h \in G$ with (5.2) and such that

$$
h \notin K_{\alpha}^{-1} B L^{-1} \cup K_{\alpha}^{-1} B L .
$$

This formula can be rephrased to say that for all $i, j \leq p$ with $i \neq j$

$$
a_{j}^{-1} a_{i} \notin h^{-1} K_{\alpha}^{-1} B
$$

and

$$
a_{j}^{-1} a_{i} \notin B^{-1} K_{\alpha} h \text {. }
$$

Put $A_{\alpha}=K_{\alpha} h \cup B$ and $h_{\alpha}=h$. Now, (1) is clear. To check (2), we compute

$$
\begin{aligned}
A_{\alpha}^{-1} A_{\alpha} & =\left(h^{-1} K_{\alpha}^{-1} \cup B^{-1}\right)\left(K_{\alpha} h \cup B\right) \\
& =h^{-1} K_{\alpha}^{-1} K_{\alpha} h \cup B^{-1} B \cup B^{-1} K_{\alpha} h \cup h^{-1} K_{\alpha}^{-1} B .
\end{aligned}
$$

For $i \neq j$, by our inductive assumption (2), we get $a_{j}^{-1} a_{i} \notin B^{-1} B$ and, by (5.2), (5.3), and (5.4), we obtain that $a_{j}^{-1} a_{i}$ does not belong to any other part of the union on the right hand side. Therefore, (2) holds for $\alpha$. 
Remark. As in Theorem 4.1(ii), Theorem 5.1(ii) can be strengthened if the group $G$ is assumed to be countable. Define

$$
\tau_{n}=\min \left(n,\left[G: G_{F C}\right]\right)
$$

and define for $v: G \rightarrow[0,1]$

$$
u_{n}(v)=\tau_{n} \inf \left\{\left(\mu_{F}\right)^{L}(v):|F| \leq n\right\} .
$$

We have $u \leq u^{T} \leq u_{n}$ for each $n$. The first inequality is obvious as is the second one if $\left[G, G_{F C}\right] \geq n$. The case $\left[G: G_{F C}\right]<n$ follows from Lemma 5.2. Now we have the following stronger version of Theorem 5.1(ii)

Let $G$ be a countable group. There exists $A \subseteq G$ such that $u^{R}(A)=1$ and $u_{n}(A) \leq 1$ for each $n$.

Note that if $\left[G: G_{F C}\right]$ is infinite, then $u_{n}(A) \leq 1$ for each $n$ implies $u(A)=0$. We leave it to the reader to modify the proof of Theorem 5.1(ii) to get the above statement.

\section{HAAR NULL SETS}

The definition of Haar null sets is contained in the introduction. A subset of a Polish group $G$ is called left Haar null if it is contained in a universally measurable set $A$ for which there exists a Borel probability measure $\mu$ on $G$ such that $\mu(g A)=0$ for all $g \in G$.

We will now apply the results proved above to answer questions of Mycielski. He asked in [18] the following two questions. Is each left Haar null subset of a Polish group Haar null? And given a Polish group $G$ and a universally measurable subset $A$ of $G$ which is not Haar null, is 1 in the interior of $A^{-1} A$ ? The answers to both these questions are in the negative. (The first of these questions was answered by Shi and Thomson [24] who produced an example in the Polish group of all homeomorphisms of the interval $[0,1]$.) In fact, we characterize all groups of the form $\prod_{n} H_{n}$ with each $H_{n}$ countable for which the answers are positive.

Theorem 6.1. Let $H_{n}, n \in \mathbb{N}$, be countable groups. The following conditions are equivalent.

(i) A subset of $\prod_{n} H_{n}$ is left Haar null if, and only if, it is Haar null.

(ii) For each universally measurable set $A \subseteq \prod_{n} H_{n}$ which is not Haar null, $A^{-1} A$ contains 1 in its interior.

(iii) For each closed set $A \subseteq \prod_{n} H_{n}$ which is not Haar null, $A^{-1} A$ is dense in some non-empty open set.

(iv) All but finitely many of the $H_{n}$ 's are FC.

Lemma 6.2. Let $H_{n}, n \in \mathbb{N}$, be countable with all but finitely many of them amenable. Let $A \subseteq \prod_{n} H_{n}$ be universally measurable. If $A$ is not left Haar null, then 1 is in the interior of $A^{-1} A$.

Proof. Let $N_{0}$ be chosen so that $H_{n}$ is amenable for $n \geq N_{0}$. Assume 1 is not in the interior of $A^{-1} A$. We can then pick a sequence $g_{m} \in \prod_{n} H_{n}$ such that $g_{m} \rightarrow 1$ 
as $m \rightarrow \infty$ and $g_{m} \notin A^{-1} A$. This last condition is equivalent to

$$
A g_{m} \cap A=\emptyset \text {. }
$$

Let $K_{n}$ be the set $\left\{g_{m}(n): m \in \mathbb{N}\right\}$. Note that since $g_{m} \rightarrow 1$, each $K_{n}$ is finite. For $n \geq N_{0}$ fix a finite set $L_{n} \subseteq H_{n}$ with the property that for all $h \in K_{n}$

$$
\left|\left(L_{n} h \backslash L_{n}\right) \cup\left(L_{n} \backslash L_{n} h\right)\right|<2^{-n}\left|L_{n}\right| \text {. }
$$

This is possible by amenability of the appropriate $H_{n}$ 's.

Consider the measure

$$
\mu=\prod_{n} \mu_{L_{n}} .
$$

Since $A$ is not left Haar null, there exists a $g \in \prod_{n} H_{n}$ with $\mu(g A)>0$. This allows us to find $N_{1} \geq N_{0}$ and $h_{n} \in H_{n}$ for $n \leq N_{1}$ such that if we let $N_{\bar{h}}=\left\{g \in \prod_{n} H_{n}\right.$ : $g(n)=h_{n}$ for $\left.n \leq N_{1}\right\}$, then

(1) $\frac{2}{3} \mu\left(N_{\bar{h}}\right)<\mu\left(g A \cap N_{\bar{h}}\right)$;

(2) $\frac{2}{3}<\prod_{n>N_{1}}\left(1-2^{-n}\right)$.

Now pick $m$ with $g_{m}(n)=1$ for all $n \leq N_{1}$. From (1) it follows that

$$
\frac{2}{3} \mu\left(N_{\bar{h}}\right)<\mu_{g_{m}}\left(g A g_{m} \cap N_{\bar{h}}\right)
$$

From (6.2) and (2) we obtain

$$
\begin{aligned}
\frac{2}{3} \mu\left(N_{\bar{h}}\right) & <\mu\left(N_{\bar{h}}\right) \prod_{n>N_{1}}\left(1-2^{-n}\right) \\
& \leq \mu\left(N_{\bar{h}}\right) \prod_{n>N_{1}} \frac{1}{\left|L_{n}\right|}\left|L_{n} \cap L_{n} g_{m}(n)^{-1}\right| \\
& =\mu\left(\prod_{n} L_{n} g_{m}(n)^{-1} \cap N_{\bar{h}}\right) \\
& =\mu_{g_{m}}\left(\prod_{n} L_{n} \cap N_{\bar{h}}\right) .
\end{aligned}
$$

Note that the support of $\mu_{g_{m}}$ is $\prod_{n} L_{n} g_{m}(n)$ and that $\mu_{g_{m}}$ is invariant under permutations of the set $\prod_{n} L_{n} g_{m}(n)$ which are products of permutations of the $L_{n} g_{m}(n)$ 's. Note also that the mapping from $\prod_{n}\left(L_{n} \cap L_{n} g_{m}(n)\right)$ to $\prod_{n} L_{n} g_{m}(n)$ given by $h \rightarrow h g_{m}$ extends to such a permutation. Therefore, we get that for any universally measurable $B \subseteq \prod_{n}\left(L_{n} \cap L_{n} g_{m}(n)\right)$,

$$
\mu_{g_{m}}(B)=\mu_{g_{m}}\left(B g_{m}\right) \text {. }
$$

From this equality and from (6.4), keeping in mind that $\prod_{n} L_{n} g_{m}(n)$ is the support of $\mu_{g_{m}}$, we get that for any universally measurable $B \subseteq \prod_{n} H_{n}$,

$$
\begin{aligned}
\mu_{g_{m}}\left(B \cap N_{\bar{h}}\right) & \leq \mu_{g_{m}}\left(B \cap \prod_{n} L_{n} \cap \prod_{n} L_{n} g_{m}(n) \cap N_{\bar{h}}\right)+\mu_{g_{m}}\left(N_{\bar{h}} \backslash \prod_{n} L_{n}\right) \\
& \leq \mu_{g_{m}}\left(\left(B \cap \prod_{n} L_{n} \cap \prod_{n} L_{n} g_{m}(n) \cap N_{\bar{h}}\right) g_{m}\right)+\left(1-\frac{2}{3}\right) \mu\left(N_{\bar{h}}\right) \\
& \leq \mu\left(B \cap N_{\bar{h}}\right)+\frac{1}{3} \mu\left(N_{\bar{h}}\right) .
\end{aligned}
$$


Applying this inequality to $B=g A g_{m}$ and combining the result with (6.3), we obtain

$$
\frac{1}{3} \mu\left(N_{\bar{h}}\right)<\mu\left(g A g_{m} \cap N_{\bar{h}}\right) .
$$

However, this inequality together with (1) yields

$$
\mu\left(g A g_{m} \cap g A\right)>0
$$

contradicting (6.1).

Proof of Theorem 6.1. (iv) $\Rightarrow(i)$ It is enough to prove (i) for universally measurable sets. Let $A \subseteq \prod_{n} H_{n}$ be universally measurable and left Haar null, and let $\mu$ be a Borel probability measure witnessing that it is left Haar null. We will find another Borel probability measure witnessing that $A$ is Haar null. Let $m_{0}$ be such that $H_{n}$ is FC if $m \geq m_{0}$. Let us also fix $0<C_{n}<1$ with

$$
\epsilon=\prod_{n} C_{n}>0 .
$$

For $n \geq m_{0}-1$ define $\pi_{n}$ and $\pi_{\infty}$ to be the projections from $\prod_{i \in \mathbb{N}} H_{i}$ to $\prod_{m_{0} \leq i \leq n} H_{i}$ and $\prod_{m_{0} \leq i} H_{i}$, respectively. (For convenience, we adopt the convention that if $n=m_{0}-1$, then $\prod_{m_{0} \leq i \leq m_{0}-1} H_{i}=\{\emptyset\}$ and $\pi_{n}$ maps all the points in its domain to $\emptyset$.) Further put

$$
\mu_{n}=\left(\pi_{n}\right)_{*} \mu \text { and } \mu_{\infty}=\left(\pi_{\infty}\right)_{*} \mu .
$$

By Lemma 5.2 for $M=1$, we obtain, for $n \geq m_{0}$, a finite nonempty set $F_{n} \subseteq H_{n}$ such that

$$
\frac{1}{C_{n}}\left(\mu_{n}\right)^{L}(v)>\left(\mu_{n-1} \times \mu_{F_{n}}\right)^{T}(v) .
$$

To make this inequality applicable to our situation, we note that it implies that for $n \geq m_{0}, v: \prod_{m_{0} \leq i \leq n} H_{i} \rightarrow[0,1]$ and for any $h \in \prod_{m_{0} \leq i \leq n} H_{i}$ with all but the $n$-th coordinate equal to 1 there exists $g \in \prod_{m_{0} \leq i \leq n} H_{i}$ with all but the $n$-th coordinate equal to 1 such that

$$
\mu_{n}\left({ }_{g} v_{h}\right)>C_{n}\left(\mu_{n-1} \times \mu_{F_{n}}\right)(v) .
$$

Inequality (6.7) is obtained by applying (6.6) to $v_{h}$. We now prove the following claim.

Claim. Let $\epsilon$ be given by (6.5). For any universally measurable $B \subseteq \prod_{m_{0} \leq i} H_{i}$, for each $h \in \prod_{m_{0} \leq i} H_{i}$ there exist $g \in \prod_{m_{0} \leq i} H_{i}$ such that for any $m \geq m_{0}$,

$$
\mu_{\infty}\left(\pi_{m}(g B h) \times H_{m+1} \times H_{m+2} \cdots\right) \geq \epsilon \cdot\left(\mu_{F_{m_{0}}} \times \mu_{F_{m_{0}+1}} \times \cdots\right)(B) .
$$

Proof of Claim. First we set up some notation. Put

$$
b=\left(\mu_{F_{m_{0}}} \times \mu_{F_{m_{0}+1}} \times \cdots\right)(B) .
$$

Let $m$ be such that $m_{0}-1 \leq m$. For $t \in \prod_{m_{0} \leq i \leq m} H_{i}$, let

$$
B_{t}=\left\{x \in \prod_{m+1 \leq i} H_{i}: t x \in B\right\}
$$


where $t x$ stands for the sequence in $\prod_{m_{0} \leq i} H_{i}$ equal to the finite sequence $t$ followed by $x$. (Here again we use the convention that $\prod_{m_{0} \leq i \leq m_{0}-1} H_{i}=\{\emptyset\}$.) Define $v_{m}: \prod_{m_{0} \leq i \leq m} H_{i} \rightarrow[0,1]$ by letting

$$
v_{m}(t)=\left(\mu_{F_{m+1}} \times \mu_{F_{m+2}} \times \cdots\right)\left(B_{t}\right) .
$$

In particular,

$$
v_{m_{0}-1}(\emptyset)=\left(\mu_{F_{m_{0}}} \times \mu_{F_{m_{0}+1}} \times \cdots\right)(B)=b .
$$

We will need the following formula connecting $v_{m}$ and $v_{m+1}$ with $m_{0}-1 \leq m$ whose verification we leave to the reader. For any $g_{i}, h_{i} \in H_{i}, m_{0} \leq i \leq m$,

$$
\begin{aligned}
\bar{g}_{m}\left(v_{m}\right)_{\bar{h}_{m}} & \left(x_{m_{0}}, \ldots, x_{m}\right) \\
= & \int_{H_{m+1}}\left(\bar{g}_{m}, 1\right) \\
& \left(v_{m+1}\right)_{\left(\bar{h}_{m}, 1\right)}\left(x_{m_{0}}, \ldots x_{m}, x\right) d \mu_{F_{m+1}}(x) .
\end{aligned}
$$

where $\bar{g}_{m}=\left(g_{m_{0}}, \ldots, g_{m}\right)$ and $\bar{h}_{m}=\left(h_{m_{0}}, \ldots, h_{m}\right)$.

We now proceed with the proof of the claim. Let us fix $h=\left(h_{m_{0}}, h_{m_{0}+1}, \cdots\right) \in$ $\prod_{m_{0}<i} H_{i}$. By induction on $m$ with $m_{0} \leq m$, we will produce $g_{m} \in H_{m}$ such that

$$
\mu_{m}\left(\bar{g}_{m}\left(v_{m}\right)_{\bar{h}_{m}}\right)>C_{m_{0}} \cdots C_{m} b
$$

where $\bar{g}_{m}=\left(g_{m_{0}}, \ldots, g_{m}\right)$ and $\bar{h}_{m}=\left(h_{m_{0}}, \ldots, h_{m}\right)$.

If we succeed and let $g=\left(g_{m_{0}}, g_{m_{0}+1}, \ldots\right)$ and $h=\left(h_{m_{0}}, h_{m_{0}+1}, \ldots\right)$, by the definition of $\epsilon$, inequality (6.10) yields our claim. Indeed, the indicator function of $\pi_{m}(g B h)$ is pointwise $\geq \bar{g}_{m}\left(v_{m}\right)_{\bar{h}_{m}}$ so, for all $m$,

$$
\begin{aligned}
\mu_{\infty}\left(\pi_{m}(g B h) \times H_{m+1} \times H_{m+2} \times \cdots\right) & \geq \mu_{m}\left(\pi_{m}(g B h)\right) \\
& \geq \mu_{m}\left(\bar{g}_{m}\left(v_{m}\right)_{\bar{h}_{m}}\right)>C_{m_{0}} \cdots C_{m} b>\epsilon b .
\end{aligned}
$$

We will show now how to find $g_{m+1}$ assuming that (6.10) holds for $\bar{g}_{m}$ and $\bar{h}_{m}$. Precisely the same argument gives (6.10) for $m=m_{0}$ if we only keep in mind that (6.8) gives

$$
\mu_{m_{0}-1}\left(v_{m_{0}-1}\right)=\left(\mu_{F_{m_{0}}} \times \mu_{F_{m_{0}+1}} \times \cdots\right)(B)=b .
$$

So assume (6.10) for $m$. From it we get

$$
\left(\mu_{m} \times \mu_{F_{m+1}}\right)\left(_{\left(\bar{g}_{m}, 1\right)}\left(v_{m+1}\right)_{\left(\bar{h}_{m}, 1\right)}\right)>C_{m_{0}} \cdots C_{m} b
$$

since by (6.9) the left hand side of the above formula is equal to the left hand side of (6.10). Now applying to it (6.7) with $v={ }_{\left(\bar{g}_{m}, 1\right)}\left(v_{m+1}\right)_{\left(\bar{h}_{m}, 1\right)}$ and $\left(1, \ldots, 1, h_{m+1}\right) \in$ $\prod_{m_{0} \leq i \leq m+1} H_{i}$, we obtain $g_{m+1} \in H_{m+1}$ such that

$$
\mu_{m+1}\left({\left(\bar{g}_{m}, g_{m+1}\right)}\left(v_{m+1}\right)_{\left(\bar{h}_{m}, h_{m+1}\right)}\right)>C_{m+1}\left(C_{m_{0}} \cdots C_{m} b\right)
$$

which is (6.10) for $m+1$ and the claim is established.

Let $\nu$ be a measure on $\prod_{i<m_{0}} H_{i}$ with a one point support. We claim that $\nu \times \mu_{F_{m_{0}}} \times \mu_{F_{m_{0}+1}} \times \cdots$ witnesses that $A$ is Haar null. If not, then for some $g, h \in \prod_{n} H_{n}$,

$$
\left(\nu \times \mu_{F_{m_{0}}} \times \mu_{F_{m_{0}+1}} \times \cdots\right)(g A h)>0 .
$$


The above inequality will remain true if we pass to a compact subset of $A$. In fact, for the sake of simplicity, we assume that $A$ is compact. Find a $t \in \prod_{i<m_{0}} H_{i}$ with

$$
\left(\mu_{F_{m_{0}}} \times \mu_{F_{m_{0}+1}} \times \cdots\right)\left(g^{\prime} A_{t} h^{\prime}\right)=\delta>0
$$

where $g^{\prime}=g\left|\left[m_{0}, \infty\right), h^{\prime}=h\right|\left[m_{0}, \infty\right)$ and $A_{t}=\left\{x \in \prod_{m_{0} \leq i} H_{i}: t x \in A\right\}$. Now from the claim, we obtain that for some $g^{\prime \prime} \in \prod_{m_{0} \leq i} H_{i}$ and for all $m \geq m_{0}$,

$$
\mu_{\infty}\left(\pi_{m}\left(g^{\prime \prime}\left(g^{\prime} A_{t} h^{\prime}\right)\left(h^{\prime}\right)^{-1}\right) \times H_{m+1} \times \cdots\right) \geq \epsilon \delta .
$$

Since $A$ is compact,

$$
g^{\prime \prime} g^{\prime} A_{t}=\bigcap_{m} \pi_{m}\left(g^{\prime \prime} g^{\prime} A_{t}\right) \times H_{m+1} \times \cdots
$$

which together with $(6.11)$ yields

$$
\mu_{\infty}\left(g^{\prime \prime} g^{\prime} A_{t}\right)>0
$$

Since $\prod_{i<m_{0}} H_{i}$ is countable, this last formula contradicts the fact that $\mu$ witnesses $A$ being left Haar null.

$(i) \Rightarrow(i v)$ Assume that infinitely many of the $H_{n}$ 's are not FC. By grouping the factor groups into finite blocks, we can assume, as we do, that none of the $H_{n}$ 's is FC. By Theorem 5.1(ii), we can find $A_{n} \subseteq H_{n}$ be such that $u\left(A_{n}\right) \leq 1 / 2$ and $u^{R}\left(A_{n}\right)=1$. Let

$$
A=\prod_{n} A_{n} .
$$

The condition $u\left(A_{n}\right) \leq 1 / 2$ implies that $A$ is left Haar null. Indeed, it gives sets $D_{n} \subseteq H_{n}, n \in \mathbb{N}$, such that $\mu_{D_{n}}\left(h A_{n}\right) \leq 1 / 2$ for all $h \in H_{n}$. Then the measure $\mu_{D_{0}} \times \mu_{D_{1}} \times \cdots$ witnesses that $A$ is left Haar null. The condition $u^{R}\left(A_{n}\right)=1$ implies that for each finite set $F \subseteq H_{n}$ there exists an $h \in H_{n}$ with $F h \subseteq A_{n}$. If $\mu$ is a Borel probability measure on $\prod_{n} H_{n}$, let $K$ be a compact set of positive measure. Then the projection $F_{n}$ of $K$ on the $n$-th coordinate is finite. By what was said above, we can find $h_{n} \in H_{n}$ with $F_{n} h_{n} \subseteq A_{n}$. If we let $h=\left(h_{n}\right)_{n} \in \prod_{n} H_{n}$, it follows that $K \subseteq A h^{-1}$; thus $\mu\left(A h^{-1}\right)>0$. Since $\mu$ was arbitrary, $A$ is not Haar null, in fact, not even right Haar null.

$(i i) \Rightarrow(i i i)$ is clear.

$($ iii $) \Rightarrow($ iv $)$ Assume that infinitely many of the $H_{n}$ 's are not FC. As in the proof of $(i) \Rightarrow(i v)$ we can suppose that all the $H_{n}$ 's are not FC. By Theorem 5.1(ii), for each $n$ we can find an $A_{n} \subseteq H_{n}$, with $u^{R}\left(A_{n}\right)=1$, for which there exists an at least two element set $D$ containing 1 such that for each $h \in H_{n}, h A_{n} \cap D$ has at most one element. This easily implies that $A_{n}^{-1} A_{n} \neq H_{n}$, in fact, $x \notin A_{n}^{-1} A_{n}$ for any $x \in D$ with $x \neq 1$. It follows that $A=\prod_{n} A_{n}$ is such that $A^{-1} A$ has dense complement in $\prod_{n} H_{n}$. Since $A^{-1} A$ is also closed, it is nowhere dense. Furthermore, $u^{R}\left(A_{n}\right)=1$ implies, as in $(i) \Rightarrow(i v)$, that $A$ is not Haar null.

$(i v) \Rightarrow(i i)$ Let $A \subseteq \prod_{n} H_{n}$ be universally measurable and not Haar null. Since the implication $(i v) \Rightarrow(i)$ has already been established, we see that $A$ is not left Haar null. Since all but finitely many of the $H_{n}$ 's are amenable, as they are FC, it follows from Lemma 6.2 that 1 is in the interior of $A^{-1} A$. 
Remark. The proof of Theorem 6.1 gives, in fact, that if infinitely many of the $H_{n}$ 's are not FC, then there exists one set $A \subseteq \prod_{n} H_{n}$ which is closed, is left Haar null, is not Haar null, and has $A^{-1} A$ nowhere dense.

\section{QUESTIONS AND ADDITIONAL REMARKS}

The first of the questions below is related to Theorem 1.1. An affirmative answer to it would show that condition $a=u$ from this theorem characterizes amenability.

Question. Is it true that if $G$ is not amenable, then, for each $\epsilon>0$, there exists a probability measure $\mu$ on $G$ with the property that for any non-empty finite set $F \subseteq G$ there exists $A \subseteq F$ such that $\left(\mu_{F}\right)(A)>1-\epsilon$ and $\mu^{L}(A)<\epsilon$ ?

I will also mention a problem related to Emerson's characterization of amenability [9] and to the measures of size of subsets of $G$ defined in the introduction: $a$, $a^{T}, u$, and $u^{T}$. Clearly all of these functions are monotonic, that is, they assign smaller values to smaller (with respect to inclusion) sets. It is natural to ask when they are subadditive, that is, when their value on $A \cup B$ is bounded by the sum of their values on $A$ and $B$. By reproducing the simple argument from [25] that Haar null sets are closed under taking finite unions, we see that $a^{T}$ is always subadditive. Since $a^{T}=u^{T}$, so is $u^{T}$. By the proof (but not the statement) of [9, Theorem 1.7 $(\mathrm{a}) \Leftrightarrow(\mathrm{b})]$, we get that $a$ is subadditive if $G$ is amenable. (To see how this proof applies here, note that $a(v)$, for a bounded $v: G \rightarrow \mathbb{R}$, is equal to inf $\|\mu * \tilde{v}\|_{\infty}$ where the infimum is taken over all $l^{1}$ non-negative functions $\mu$ on $G$ with the $l^{1}$ norm 1 and $\tilde{v}(g)=v\left(g^{-1}\right)$. With this in mind, we see that $[9$, Theorem $1.7,(\mathrm{a}) \Leftrightarrow(\mathrm{b})]$ says that $G$ is amenable precisely when $a\left(v_{1}\right)=a\left(v_{2}\right)=0$ implies $a\left(v_{1}+v_{2}\right)=0$ for bounded functions $v_{1}, v_{2}$.) Since $a=u$ on amenable groups, $u$ is subadditive on such groups as well. The following question seems, therefore, natural. An affirmative answer to it would give a characterization of amenability.

Question. Is it true that if $a$ (or $u$ ) is subadditive as a function of subsets of a group $G$, then $G$ is amenable?

Acknowledgement. I thank the referee for useful comments particularly those leading to simplification of the proof of Lemma 3.6.

\section{REFERENCES}

[1] H. Becker and A.S. Kechris, The Descriptive Set Theory of Polish Group Actions, London Mathematical Society Lecture Notes Series 232, Cambridge University Press, 1996.

[2] Y. Benyamini and J. Lindenstrauss, Geometric Nonlinear Functional Analysis, Colloquium Publications, American Mathematical Society, 2000.

[3] P. Billingsley, Probability and Measure, Wiley, 1995.

[4] J.P.R. Christensen, On sets of Haar measure zero in Abelian Polish groups, Israel J. Math. 13(1972), 255-260.

[5] J.P.R. Christensen, Topology and Borel Structure, North-Holland, 1974.

[6] M.M. Day, Amenable semigroups, Illinois J. Math. 1(1957), 509-544.

[7] M.M. Day, Lumpy subsets in left-amenable, locally compact semigroups, Pacific J. Math. 62(1976), 87-92. 
[8] M.M. Day, Left thick to left lumpy — a guided tour, Pacific. J. Math. 101(1982), 71-92.

[9] W.R. Emerson, Characterizations of amenable groups, Trans. Amer. Math. Soc. 241(1978), 183-194.

[10] J. Erdős, The theory of groups with finite classes of conjugate elements, Acta Math. Acad. Sci. Hung. 5(1954), 45-58.

[11] E. Hewitt and K.A. Ross, Abstract Harmonic Analysis, Vol. I, Springer Verlag, 1963.

[12] B.R. Hunt, T. Saur, and J.A. Yorke, Prevalence: a translation invariant "almost every" on infinite dimensional spaces, Bull. Amer. Math. Soc. (N.S.) 27(1992), 217-238.

[13] R.V. Kadison and J.R. Ringrose, Fundamentals of the Theory of Operator Algebras, Vol. II, Academic Press, 1986.

[14] J. Lindenstrauss, E. Matouškova, and D. Preiss, Lipschitz image of a measure-null set can have a null complement, Israel J. Math. 118(2000), 207-219.

[15] J. Lindenstrauss and D. Preiss, On Frechet differentiability of Lipschitz maps between Banach spaces, Ann. of Math. 157(2003), 257-288.

[16] E. Matouškova, Translating finite sets into convex sets, Bull. London Math. Soc. 33(2001), $711-714$.

[17] T. Mitchell, Constant functions and left invariant means on semigroups, Trans. Amer. Math. Soc. 119(1965), 244-261.

[18] J. Mycielski, Some unsolved problems on the prevalence of ergodicity, instability, and algebraic independence, Ulam Quarterly 1(1992), 30-37.

[19] B.H. Neumann, Groups with finite conjugacy classes, Proc. London Math. Soc. 1(1951), $178-187$.

[20] B.H. Neumann, Groups covered by finitely many cosets, Publ. Math. Debrecen 3(1954), 227242.

[21] A.L.T. Paterson, Amenable groups for which every topological left invariant mean is invariant, Pacific J. Math. 84(1979), 391-397.

[22] A.L.T. Paterson, Amenablity, Mathematical Surveys and Monographs 29, Amer. Math. Soc., 1988.

[23] J.-P. Pier, Amenable Locally Compact Groups, Wiley, 1984.

[24] H. Shi and B.S. Thomson, Haar null sets in the space of automorphisms of $[0,1]$, Real Anal. Exchange 24(1998/99), 337-350.

[25] F. Topsøe and J. Hoffman-Jørgensen, Analytic spaces and their applications, in Analytic Sets, eds. C.A. Rogers et. al., Academic Press (1980), pp. 317-401.

Department of Mathematics, University of Illinois, 1409 W. Green St., Urbana, IL 61801

E-mail address: ssolecki@math.uiuc.edu 